Edition KWV 
Die „Edition KWV“ beinhaltet hochwertige Werke aus dem Bereich der Wirtschaftswissenschaften. Alle Werke in der Reihe erschienen ursprünglich im Kölner Wissenschaftsverlag, dessen Programm Springer Gabler 2018 übernommen hat.

Weitere Bände in der Reihe http://www.springer.com/series/16033 
Karsten Schütz

\section{Die Nutzung von Paneldaten im strategischen Marketing von Fast Moving Consumer Goods-Herstellern}

Springer Gabler 
Karsten Schütz

Wiesbaden, Deutschland

Bis 2018 erschien der Titel im Kölner Wissenschaftsverlag, Köln

Dissertation Universität zu Köln, 2006

\section{Edition KWV}

ISBN 978-3-658-24689-1 ISBN 978-3-658-24690-7 (eBook)

https://doi.org/10.1007/978-3-658-24690-7

Die Deutsche Nationalbibliothek verzeichnet diese Publikation in der Deutschen Nationalbibliografie; detaillierte bibliografische Daten sind im Internet über http://dnb.d-nb.de abrufbar.

\section{Springer Gabler}

(C) Springer Fachmedien Wiesbaden GmbH, ein Teil von Springer Nature 2007, Nachdruck 2019

Ursprünglich erschienen bei Kölner Wissenschaftsverlag, Köln, 2007

Das Werk einschließlich aller seiner Teile ist urheberrechtlich geschützt. Jede Verwertung, die nicht ausdrücklich vom Urheberrechtsgesetz zugelassen ist, bedarf der vorherigen Zustimmung des Verlags. Das gilt insbesondere für Vervielfältigungen, Bearbeitungen, Übersetzungen, Mikroverfilmungen und die Einspeicherung und Verarbeitung in elektronischen Systemen.

Die Wiedergabe von allgemein beschreibenden Bezeichnungen, Marken, Unternehmensnamen etc. in diesem Werk bedeutet nicht, dass diese frei durch jedermann benutzt werden dürfen. Die Berechtigung zur Benutzung unterliegt, auch ohne gesonderten Hinweis hierzu, den Regeln des Markenrechts. Die Rechte des jeweiligen Zeicheninhabers sind zu beachten.

Der Verlag, die Autoren und die Herausgeber gehen davon aus, dass die Angaben und Informationen in diesem Werk zum Zeitpunkt der Veröffentlichung vollständig und korrekt sind. Weder der Verlag, noch die Autoren oder die Herausgeber übernehmen, ausdrücklich oder implizit, Gewähr für den Inhalt des Werkes, etwaige Fehler oder Äußerungen. Der Verlag bleibt im Hinblick auf geografische Zuordnungen und Gebietsbezeichnungen in veröffentlichten Karten und Institutionsadressen neutral.

Springer Gabler ist ein Imprint der eingetragenen Gesellschaft Springer Fachmedien Wiesbaden GmbH und ist ein Teil von Springer Nature

Die Anschrift der Gesellschaft ist: Abraham-Lincoln-Str. 46, 65189 Wiesbaden, Germany 
Et es wie et es.

Et kütt wie et kütt.

Et hätt noch immer joot jejange.

(Art. 1 - 3 des Kölschen Grundgesetzes

mit direktem Bezug zur strategischen Marketingplanung) 


\section{GELEITWORT}

Paneldaten stellen eine wichtige, in der Praxis viel genutzte Informationsquelle für Marketing- und Vertriebsentscheidungen dar. Sie werden allerdings überwiegend für verhältnismäßig kurzfristige, operative Fragestellungen herangezogen, und dem entsprechen auch die Informationsangebote der Marktforschungsinstitute, die Panelstudien durchführen. In der einschlägigen betriebswirtschaftlichen Fachliteratur stehen ebenfalls die operativen Auswertungsmöglichkeiten von Paneluntersuchungen im Vordergrund. Analysen mit Blick auf die Sicherung langfristiger Erfolgspotenziale durch panelgestützte Marketingstrategien klingen zwar in manchen veröffentlichten Arbeiten an, werden aber nicht im Rahmen einer umfassenden Systematik erörtert.

Herr Dr. Schütz hat sich die Aufgabe gestellt, die möglichen Beiträge von Panels zur Fundierung strategischer Marketingentscheidungen systematisch aufzuzeigen, wobei er sich auf Daten aus Verbraucher- und Handelspanels mit Bezug auf sog. Fast Moving Consumer Goods (FMCG) konzentriert.

Zu diesem Zweck skizziert der Autor zunächst im Überblick die inhaltlichen Teilgebiete der Marketingplanung und ordnet sie der strategischen Perspektive (Schaffung und Erhaltung von Erfolgspotenzialen) bzw. der operativen Sichtweise (Ausschöpfung der gegebenen Potenziale) zu. Hieraus ergibt sich eine Gliederung des Buches in fünf Hauptteile, die sich mit Panelinformationen für die strategisch ausgerichtete Situationsanalyse, Früherkennung und Langfristprognose beschäftigen sowie mit der Wahl künftiger Produkt-MarktKombinationen („Defining the Business“) und mit mehrperiodig angelegten Marketing-Mix-Konzepten.

In den Erörterungen zur Situationsanalyse mit strategischen Implikationen geht Herr Dr. Schütz ausführlich auf Kundenstruktur-, Programmstruktur-, Distributions- und Konkurrentenanalysen ein. Unter den dargestellten Merkmalen der Kundenstruktur erscheinen z.B. Angaben zur Entwicklung der Altersklassen im Kundenkreis und zum Familien-Lebenszyklus sowie Kundenportfolios strategisch aufschlussreich. 
Für die Beurteilung der Programmstruktur im Hinblick auf Erfolgspotenziale wird u.a. den Informationen zur Phasenbestimmung im Produktlebenszyklus und zur Erstellung von Produktportfolios Bedeutung beigemessen. Portfoliobetrachtungen spielen auch im Abschnitt zur Distributionsanalyse eine Rolle, ebenso aber die Zielgruppenausrichtung der Distribution sowie Kenngrößen zur Bestimmung der relativen Machtposition in den HerstellerHandels-Beziehungen.

Bei der Konkurrentenanalyse diskutiert Herr Dr. Schütz, inwieweit Paneldaten zur Identifikation der relevanten Wettbewerber, ihrer Kunden- und Produktprogrammstruktur sowie ihrer Marketing-Mix-Gestaltung geeignet sind.

Die panelgestützte strategische Früherkennung wird kurz skizziert.

Herr Dr. Schütz weist auf Indikatoren bezüglich des Produktprogramms wie auch auf abnehmer-, handels- und konkurrenzorientierte Indikatoren hin, die frühzeitige Hinweise auf Bedrohungen oder günstige Gelegenheiten anzeigen.

Im Abschnitt über strategische Prognosen auf der Grundlage von Paneldaten verdient besonders die längerfristige Vorhersage mithilfe der Kohortenanalyse Aufmerksamkeit. Sie ist besser fundiert als z.B. ausschließlich auf demografische Daten der Altersstruktur von Konsumenten gestützte Projektionen. Sie kombiniert altersspezifische Faktoren, Einflüsse periodenbedingter Umfeldgegebenheiten und die Zugehörigkeit $\mathrm{zu}$ einer bestimmten „Generation“ (Kohorte) des Kauf- und Konsumverhaltens. Die aus der Verknüpfung von Alters-, Perioden- und Kohorteneffekten entstehende Konfundierungsproblematik wird von Herrn Dr. Schütz nicht übersehen. In diesem Kapitel wird gut verdeutlicht, dass Paneldaten eine hilfreiche Grundlage für Kohortenanalysen bilden und dass sich hiermit Vorausschätzungen für die strategische Beurteilung von Erfolgspotenzialen gewinnen lassen.

Ebenso aufschlussreich ist der Abschnitt der Arbeit, der sich mit dem so genannten „Defining the Business" beschäftigt. Es geht dabei um die systematische Suche künftiger Produkt-, Markt- und Technologiekombinationen, die wirtschaftlichen Erfolg versprechen. Inzwischen finden sich in der Literatur viele Beispiele zur Illustration dieses strategischen 
Planungsansatzes. Bisher ist aber noch nicht versucht worden, für diese scheinbar typisch qualitative Wahl der künftigen Geschäftstätigkeit Paneldaten heranzuziehen.

Herr Dr. Schütz zeigt in anschaulicher Weise anhand von Datensätzen der GfK auf, dass sich mithilfe der Assoziationsanalyse Muster im Kaufverhalten bestimmter Verbrauchersegmente aufdecken lassen, aus denen sich Anregungen für die künftige Produktprogrammpolitik sowie für deren gezielte Ausrichtung auf Konsumentengruppen und die von diesen gewünschten Nutzenbündel ergeben. Die Assoziationsanalyse ist ein Verfahren des sog. Data Mining, das z.B. zur Aufdeckung von Verbundbeziehungen beim Kaufverhalten bzw. Verwenderbedarf beiträgt. Herr Dr. Schütz geht im Anschluss an seine konkreten Anwendungsbeispiele auch auf die Frage ein, wie sich so ermittelte potenzielle Felder der künftigen Geschäftstätigkeit auf ihre wirtschaftliche Ergiebigkeit beurteilen lassen (Merkmale der Nachfrager, der Distribution und der Konkurrenzstruktur).

Sehr komprimiert wird abschließend auf strategische Gesichtspunkte des Marketing-Mix hingewiesen. Dass dieser Teil der Arbeit relativ knapp gehalten ist, hat mehrere Gründe. Viele Entscheidungen zur Gestaltung des MarketingMix sind qualitativer Art, so vor allem in der Produkt- und Kommunikationspolitik, wofür geeignete Paneldaten fehlen. Zudem sind manche Folgerungen für Marketing-Mix-Konzepte schon im Kapitel zum Defining the Business angesprochen worden.

Insgesamt hat sich Herr Dr. Schütz mit einer Thematik auseinander gesetzt, die unter dem Blickwinkel strategischer Marketingentscheidungen bislang noch nicht umfassend aufgearbeitet worden ist. Er gibt in systematischer Weise einen Überblick, der auch zu weiteren Forschungen auf diesem Gebiet anregen kann.

Köln, im November 2006

Prof. Dr. Dr. h.c. Richard Köhler Emeritus am Marketing-Seminar der Universität zu Köln 


\section{VORWORT}

Die vorliegende Arbeit wurde im Sommer 2006 von der Wirtschafts- und Sozialwissenschaftlichen Fakultät der Universität zu Köln als Dissertation angenommen. Sie entstand zu großen Teilen während meiner Zeit als Wissenschaftlicher Mitarbeiter am Seminar für Marktforschung und Marketing und beschäftigt sich mit der Frage, wie Hersteller sog. ,Fast Moving Consumer Goods“ Paneldaten, welche von großen Marktforschungsinstituten erhoben werden, für strategische Fragestellungen in der Marketing-Planung nutzen können.

Für ihre Unterstützung und ihren Beitrag zum Gelingen dieser Arbeit möchte ich folgenden Personen ganz besonders danken: Meinem Doktorvater, Herrn Prof. Dr. Dr. h.c. Richard Köhler für seine fachliche und interessierte Unterstützung und die hierbei gewährten Freiräume. Für die Übernahme des Zweitgutachtens danke ich Herrn Prof. Dr. Müller-Hagedorn sehr herzlich. Darüber hinaus bin ich der GfK Nürnberg zu großem Dank verpflichtet, die mir reale Paneldaten zur Verfügung gestellt hat. In diesem Zusammenhang möchte ich insbesondere Herrn Dr. Raimund Wildner meinen Dank aussprechen, der mein Hauptansprechpartner bei der GfK war und mich auch bei schwierigen Fragestellungen stets mit großer Hilfsbereitschaft unterstützt hat.

Großen Dank möchte ich zudem meinen ehemaligen Kollegen am MarketingSeminar aussprechen. Dies gilt insbesondere für Herrn Prof. Dr. Axel Faix, mit dem ich eine Vielzahl fruchtbarer fachlicher Diskussionen geführt habe, die häufig zu einer klareren Sicht der Dinge geführt haben, was im Rahmen eines Dissertationsprojektes von enormem Wert ist. Ebenfalls sehr dankbar bin ich Herrn Dr. Frank Schlein, der sich mit großer Sorgfalt und Einsatzbereitschaft der Durchsicht meiner Arbeit gewidmet hat, obwohl er sich schon im Berufsleben befand. Allen ehemaligen Mitarbeitern zusammen möchte ich für die sehr schöne Zeit am Seminar danken, die nicht unbedingt immer durch Arbeit, sondern auch durch außeruniversitäre Aktivitäten gekennzeichnet war. Letztere verlängerten zwar die Promotionsdauer, verschönerten aber in überproportionalem Maße das Leben in dieser Zeit. 
Mein größter Dank gilt meinen Eltern. Sie standen mir jederzeit mit Rat und Tat zur Seite, haben mich immer gefördert und mir in allen Situationen des Lebens die nötige Gelassenheit vermittelt. Ohne sie wäre meine Ausbildung in dieser Form nicht möglich gewesen. Auch meiner Freundin Eva Müller möchte ich danken, und zwar sowohl für die Zeit, in der sie mich vom Schreiben der Dissertation abhielt als auch für die Zeit, in der sie dies nicht tat.

Rückblickend kann ich zu meinem Promotionsprojekt mit all seinen Facetten nur sagen, dass ich es jederzeit wieder tun würde.

Köln, im November 2006

Karsten Schütz 


\section{Inhaltsverzeichnis}

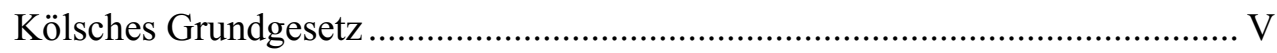

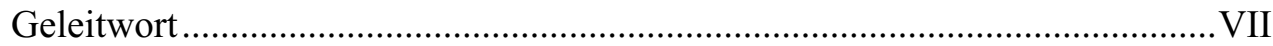

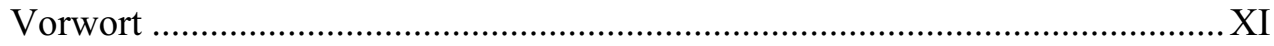

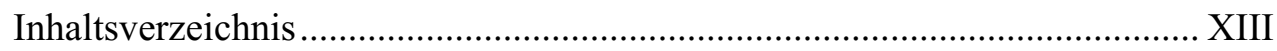

Abbildungsverzeichnis ............................................................................. XIX

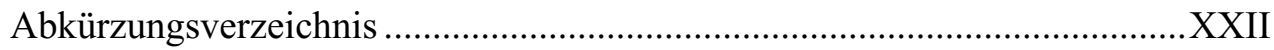

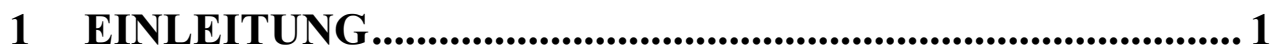

1.1 Problemstellung und Zielsetzung der Arbeit ............................................ 1

1.2 Aufbau der Arbeit............................................................................... 2

2 GRUNDLAGEN DES STRATEGISCHEN MARKETING UND DER PANELMETHODE ................................................5

2.1 Strategisches Marketing ................................................................................ 5

2.1.1 Strategische und operative Marketing-Planung .................................... 5

2.1.2 Teilaufgaben der Marketing-Planung …............................................. 7

2.2 Grundlegende Anforderungen an Informationen zur

Unterstuitzung strategischer Marketing-Entscheidungen ................... 12

2.3 Das Panel als standardisiertes Erhebungsinstrument der

Marktforschung ..................................................................................................... 14

2.3.1 Konstitutive Merkmale eines Panels.................................................... 14

2.3.2 Grundsätzliche Informationsinhalte von Panels und Abgrenzung gegenüber ähnlichen Erhebungsmethoden .......................................... 17

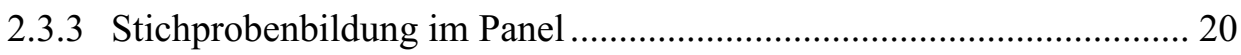

2.3.4 Probleme von Panelerhebungen.......................................................... 24

2.3.4.1 Stichprobenbezogene Probleme...................................................... 24

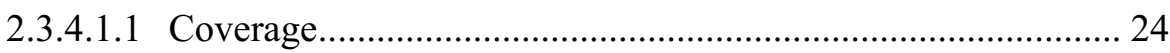

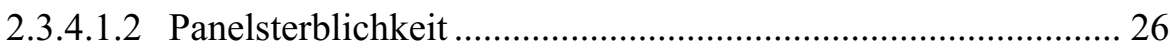

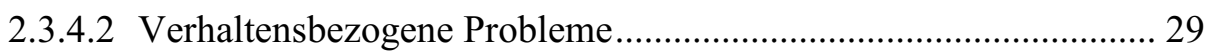

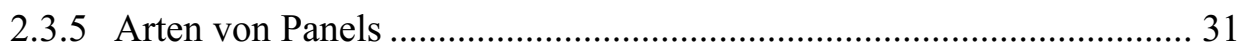

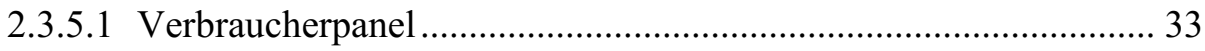


2.3.5.1.1 Erhebungseinheiten des Verbraucherpanels ........................... 33

2.3.5.1.2 Erhebungsgegenstände des Verbraucherpanels ....................... 35

2.3.5.1.3 Erhebungsmethoden des Verbraucherpanels ........................... 40

2.3.5.2 Handelspanel.......................................................................... 43

2.3.5.2.1 Erhebungseinheiten des Handelspanels ................................. 43

2.3.5.2.2 Erhebungsgegenstände des Handelspanels .............................. 44

2.3.5.2.3 Erhebungsmethoden des Handelspanels .................................. 45

3 SITUATIONSANALYSE ......................................................... 47

3.1 Kundenstrukturanalyse.................................................................... 48

3.1.1 Abgrenzung der Betrachtungsebene .................................................. 48

3.1.2 Erfassung strategisch relevanter Merkmale der Kundenstruktur.......... 49

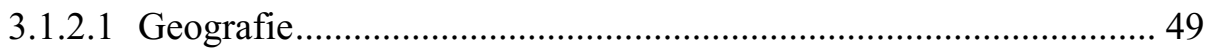

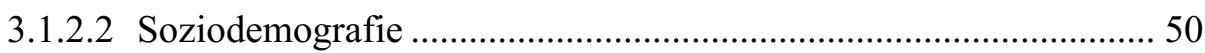

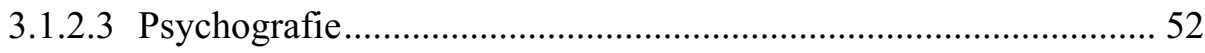

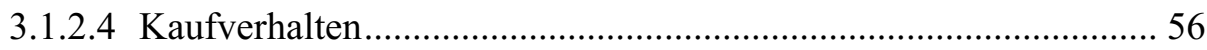

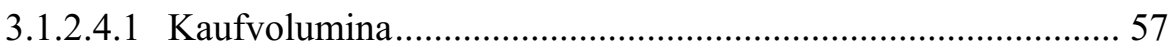

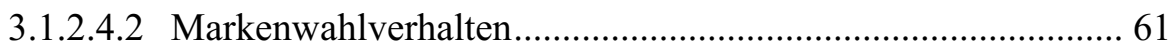

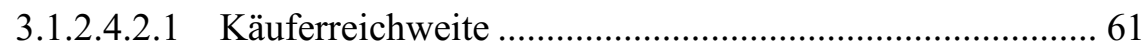

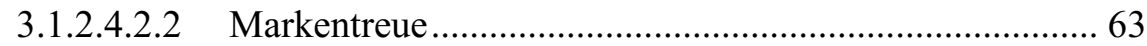

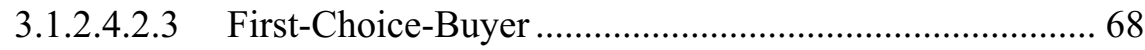

3.1.2.4.2.4 Unterteilung der Kunden nach Shopper-Typen .............. 70

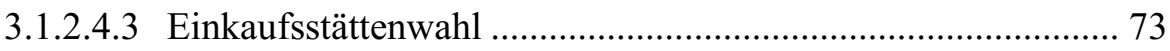

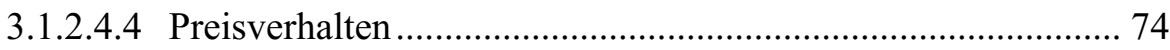

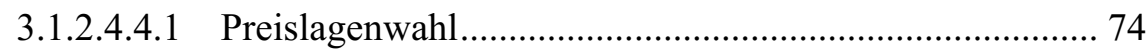

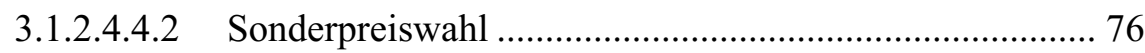

3.1.3 Kennzahlen, ABC-Analysen und Kundenportfolios als Möglichkeiten zur Verdichtung kundenstrukturbezogener

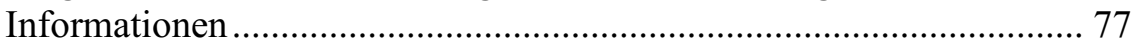

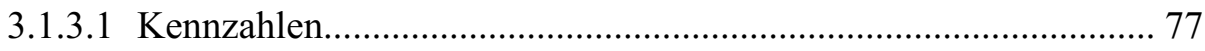

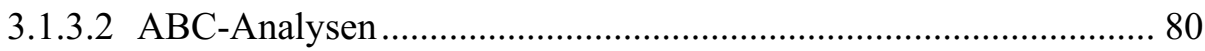


3.1.3.3 Kundenportfolios 81

3.2 Programmstrukturanalyse ................................................................90

3.2.1 Absatzstruktur des Leistungsprogramms .......................................... 90

3.2.2 „Brand Health“ als besonderes Merkmal der Absatzstruktur................ 92

3.2.3 Produktlebenszyklusbasierte Altersstruktur des

Leistungsprogramms

3.2.3.1 Möglichkeiten der Phasenabgrenzung im Rahmen des Produktlebenszyklus ................................................................ 100

3.2.3.1.1 Absatzbezogene Phasenabgrenzung …………...................... 100

3.2.3.1.2 Kundenstrukturbezogene Phasenabgrenzung ........................ 102

3.2.3.2 Die Produktinnovationsrate als besonderes Merkmal der Altersstruktur

3.2.4 Gesamtsicht des Leistungsprogramms mit Hilfe der Portfolioanalyse. 106

3.3 Distributionsanalyse

3.3.1 Erfassung des Distributionsumfangs und der

Distributionskonzentration

3.3.2 Grundsätzliche Produktprogramm- und Zielgruppenadäquanz der Distribution

3.3.3 Preisstellung der Händler 113

3.3.3.1 Generelles Preisniveau.. 114

3.3.3.2 Häufigkeit von Sonderpreisaktionen 115

3.3.4 Distributions-Portfolios 116

3.3.5 Die Bedeutung und Ermittlung der Machtposition des Herstellers im Absatzkanal

3.3.5.1 Umsatzrelation zwischen Hersteller und Händler 121

3.3.5.2 Handelsmarkenanteil im Sortiment des Händlers. 124

3.3.5.3 Breite der Produktpalette des Herstellers. 125

3.4 Konkurrentenanalyse ........................................................................ 126

3.4.1 Identifikation relevanter Konkurrenten.......................................... 128

3.4.1.1 Leistungsprogrammbezogene Identifikation von Konkurrenten.. 129 
3.4.1.2 Nachfragerbezogene Identifikation von Konkurrenten

3.4.2 Kundenbezogene Konkurrentenanalyse............................................ 132

3.4.3 Produktbezogene Konkurrentenanalyse............................................. 138

3.4.4 Verhaltensbezogene Konkurrentenanalyse ......................................... 143

3.4.4.1 Preisbezogene Konkurrentenanalyse ......................................... 143

3.4.4.1.1 Kreuzpreis-Elastizität der Nachfrage .................................... 143

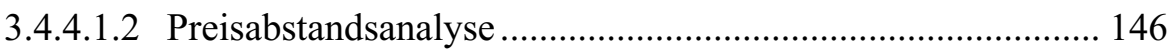

3.4.4.2 Distributionsbezogene Konkurrentenanalyse .............................. 147

3.4.5 Zusammenfassende Diagnose der Wettbewerbssituation................... 149

4 PANELDATENGESTÜTZTE STRATEGISCHE FRÜHERKENNUNG

4.1 Entwicklungslinien der Früherkennung und Typen von

Früherkennungsinformationen ................................................................... 153

4.2 Eignung von Panels für die Früherkennung aufgrund ihrer spezifischen Eigenschaften ............................................................................... 157

4.2.1 Umfang der Datenbereitstellung ...................................................... 157

4.2.2 Möglichkeit der Datendisaggregation ................................................. 159

4.2.3 Datengewinnung auf individueller Ebene.......................................... 160

4.2.4 Langfristigkeit und Kontinuität der Datenerhebung ........................... 162

4.3 Möglichkeiten der Generierung von

Früherkennungsinformationen auf Paneldatenbasis........................ 163

4.3.1 Abweichungsanalyse geplanter Zielgrößen ...................................... 163

4.3.2 Früherkennung auf der Basis von Vorlaufindikatoren ....................... 169

5 STRATEGISCHE PROGNOSEN AUF

PANELDATENBASIS ...................................................... 177

5.1 Abgrenzung von Prognoseverfahren und ihre Relevanz für strategische Fragestellungen.......................................................................... 177

5.2 Kurz- bis mittelfristige Absatzprognosen mittels des ParfittCollins-Ansatzes ...................................................................................... 180

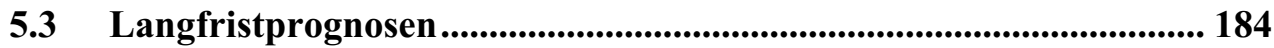

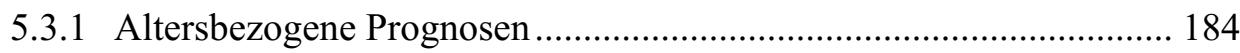


5.3.2 Kohortenanalytische Prognosen auf der Basis von Paneldaten 187

5.3.2.1 Allgemeine Darstellung der Kohortenanalyse

5.3.2.1.1 Alters-, Kohorten- und Periodeneffekte als Ursachen von

Veränderungen in Populationen

5.3.2.1.2 Die Erfassung von Alters-, Kohorten- und Periodeneffekten in der Standardkohortentabelle

5.3.2.1.3 Die Konfundierungsproblematik der Kohortenanalyse und Ansätze zu deren Lösung

5.3.2.2 Prognosen auf der Grundlage der Kohortenanalyse 200

5.3.2.3 Unterstützung der Szenariotechnik durch Kohortenanalysen ...... 204

5.3.2.4 Eignung von Panels als Datenbasis für Kohortenanalysen 205

6 DEFINING THE BUSINESS 211

6.1 Der dreidimensionale Suchrahmen von Abell 211

6.2 Aufgabenkomplexe im Defining the Business 212

6.3 Einsatzmöglichkeiten von Paneldaten im Rahmen der Aufgabenkomplexe des Defining the Business.

6.3.1 Nutzung von Paneldaten bei der Segmentierung des dreidimensionalen Suchraumes.

6.3.1.1 Segmentierung der Abnehmerachse ........................................... 215

6.3.1.2 Segmentierung der Technologieachse ........................................ 216

6.3.1.3 Segmentierung der Funktionsachse ............................................ 217

6.3.1.4 Exemplarische Konkretisierung eines Abell-Suchrahmens am Beispiel eines Getränkeherstellers

6.3.2 Suche und Identifikation potenzieller Produkt-MarktKombinationen

6.3.2.1 Ableitung von Suchstrategien

6.3.2.2 (Produkt-)Technologiedimension als Ankerpunkt der Suchaktivitäten.

6.3.2.3 Nachfragerdimension als Ankerpunkt der Suchaktivitäten 224

6.3.2.4 Funktionsdimension als Ankerpunkt der Suchaktivitäten 226 
6.3.2.5 Möglichkeiten der methodischen Unterstützung der Suche und Identifikation potenzieller Produkt-Markt-Kombinationen mit

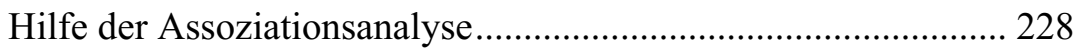

6.3.2.5.1 Grundlagen der Assoziationsanalyse .................................... 228

6.3.2.5.1.1 Aufdeckung von Zusammenhängen mittels der Assoziationsanalyse..................................................... 228

6.3.2.5.1.2 Regelgenerierung und Gütemaße der

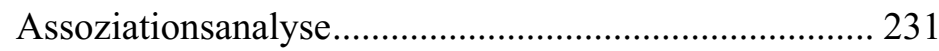

6.3.2.5.2 Anwendungsbeispiele ........................................................ 236

6.3.2.5.2.1 Analyse des Kaffeemarktes .......................................... 237

6.3.2.5.2.2 Warengruppenübergreifende Analyse........................... 246

6.3.3 Bewertung und Auswahl der Produkt-Markt-Kombinationen............ 250

6.3.3.1 Merkmale der Nachfrager ........................................................ 251

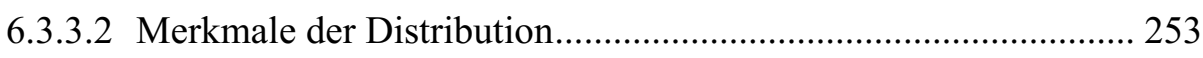

6.3.3.3 Merkmale der Konkurrenzstruktur ............................................ 254

7 STRATEGISCHER MARKETING-MIX............................. 259

7.1 Strategische Produktpolitik ................................................................ 260

7.2 Strategische Preispolitik................................................................ 261

7.3 Strategische Distributionspolitik .................................................................. 263

7.4 Strategische Kommunikationspolitik.................................................... 266

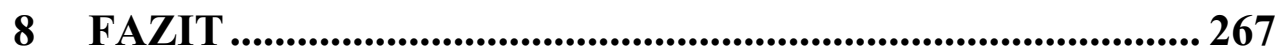

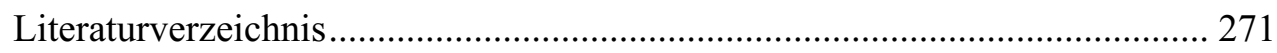




\section{Abbildungsverzeichnis}

Abb. 1: Aufgaben der Marketing-Planung ……........................................... 8

Abb. 2: Coverage des Verbraucher- und Handelspanels ............................... 26

Abb. 3: Durchgehende Masse im Jahr 2003 .................................................. 29

Abb. 4: Grundsätzliche Arten von Panels........................................................ 33

Abb. 5: Dimensionen einer Panelzahl.......................................................... 36

Abb. 6: Artikelpyramide am Beispiel des Warengruppenkorbs „Alkoholische Getränke“............................................................. 37

Abb. 7: Inhalte der jährlich durchgeführten Standardeinfragen ................... 38

Abb. 8: Datenstruktur des Verbraucherpanels .............................................. 40

Abb. 9: Kaufintensitäten für eine bestimmte Sorte Bohnenkaffee............... 59

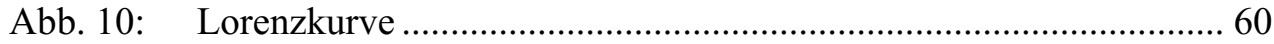

Abb. 11: Käuferreichweiten unterschiedlicher (Dach-)Marken in Prozent aller Haushalte in 2003

Abb. 12: Bedarfsdeckungsklassen für zwei Marken ..................................... 66

Abb. 13: Bedarfsdeckungsraten im Zeitablauf bei einer sinkenden

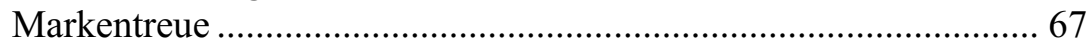

Abb. 14: First Choice Buyer der Marken A und B ........................................... 69

Abb. 15: Umsatzbedeutung First Choice Buyer ........................................ 70

Abb. 16: Shopper-Typen in Deutschland im Jahr 1999 ................................ 72

Abb. 17: Monofaktorielles Kundenportfolio ................................................. 85

Abb. 18: Monofaktorielles Kundenportfolio mit Visualisierung der

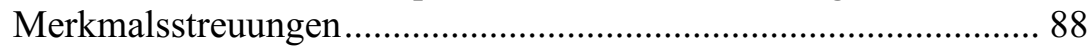

Abb. 19: Bedarfsintensität-Markentreue-Portfolio ........................................ 89

Abb. 20: Komponenten der „Brand Health“................................................. 93

Abb. 21: Bedeutung der unterschiedlichen Shopper-Typen nach

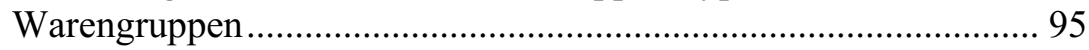

Abb. 22: Schematische Darstellung der Promotion-Falle ............................... 96

Abb. 23: (Produkt-)Lebenszyklus .................................................................... 98 
Abb. 24: Adoptergruppen und ihre relativen Übernahmezeiten von Innovationen

Abb. 25: Ableitung eines Portfolios aus Paneldaten ..................................... 107

Abb. 26: Distributionsportfolio................................................................... 117

Abb. 27: Absatzmittleranalyse bezüglich der Preisdurchsetzbarkeit für ein bestimmtes Produkt

Abb. 28: Absatzwege-Abhängigkeits-Matrix

Abb. 29: Teilaufgaben der Konkurrentenanalyse im „Strategischen Dreieck“

Abb. 30: Nebeneinanderverwendungsanalyse

Abb. 31: Käuferwanderung - Analyse der Zu- und Abwanderer.

Abb. 32: Käuferwanderung - Analyse der Wiederkäufer 136

Abb. 33: Gain\&Loss-Innenmatrix 139

Abb. 34: Gain\&Loss-Analyse 142

Abb. 35: Preisabstandsanalyse

Abb. 36: Distributionsüberschneidungsanalyse. 148

Abb. 37: Stärken-Schwächen-Profil

Abb. 38: Das Trajektorien-Konzept in der Zielplanung. 165

Abb. 39: Trajektorien-Bündel einer disaggregierten Gap-Analys 168

Abb. 40: Idealverlauf eines Indikators und der Zielvariablen 169

Abb. 41: Früherkennungsindikatoren auf unterschiedlichen Analyseebenen

Abb. 42: Entwicklung der Penetration eines Neuproduktes A 181

Abb. 43: Berechnung des Wiederkaufverhaltens seit dem Erstkauf 182

Abb. 44: Standardkohortentabelle mit hypothetischen Daten zur Käuferreichweite (in \%).

Abb. 45: Realisierbare Messungen im Rahmen des Kohortendesigns......... 192

Abb. 46: Reiner Alterseffekt........................................................................... 195

Abb. 47: Reiner Periodeneffekt ...................................................................... 195

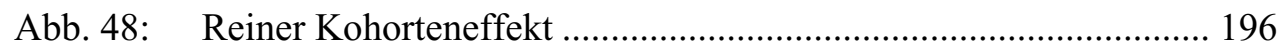

Abb. 49: Szenarioanalyse auf der Grundlage kohortenanalytischer Überlegungen 
Abb. 50: Dreidimensionaler Suchrahmen nach Abell

Abb. 51: Mögliche Konkretisierung eines Suchraums für einen Getränkehersteller auf Paneldatenbasis

Abb. 52: Mögliche Suchstrategien............................................................ 220

Abb. 53: Abellscher Suchrahmen mit Leistungsangebot als Ankerpunkt.... 223

Abb. 54: Abellscher Suchrahmen mit Leistungsangebot als Ankerpunkt und Variation der Nachfragersektoren und Funktionsanforderungen

Abb. 55: Abellscher Suchrahmen mit Nachfragergruppe als Ankerpunkt... 225

Abb. 56: Abellscher Suchrahmen für einen Hersteller von

Molkereiprodukten mit Funktionserfüllung als Ankerpunkt.

Abb. 57: Mögliche Ereigniskombinationen bei unterschiedlichen

Transaktionen in einer Datenbasis $D$

Abb. 58: Erfasste Merkmale des Kaffee-Datensatzes im Zeitraum vom $01.01 .2002-31.12 .2002$

Abb. 59: Assoziationsregeln Warengruppe Kaffee (supmin(präm): 3,5\%; confmin: $10 \%$ ).

Abb. 60: Assoziationsregeln Warengruppe Kaffee (supmin(präm): 0,6\%; confmin: $10 \%$ ).

Abb. 61: Assoziationsregeln Warengruppe Kaffee (supmin(präm): 0,6\%; confmin: $5 \%$ ).

Abb. 62: Assoziationsregeln Warengruppe Kaffee (supmin(präm): 0,6\%; confmin: $5 \%$ ).

Abb. 63: Assoziationsregeln Warengruppe Kaffee (supmin(präm): 0,6 \%; confmin: $10 \%$ ).

Abb. 64: Assoziationsregeln Warengruppe Nudeln (supmin(präm): $2 \%$; confmin: $20 \%$ ).

Abb. 65: Reaktionszeiten auf die Einführung eines Neuproduktes 256

Abb. 66: Idealtypische Formen der Skimming- und Penetrationspreisstrategie 262

Abb. 67: Entfernungsabhängige Umsatzanteile als Maß für die Attraktion unterschiedlicher Absatzmittler. 264

Abb. 68: Warengruppenspezifische Attraktion unterschiedlicher Absatzmittler. 


\section{Abkürzungsverzeichnis}

\begin{tabular}{lll} 
Abb. & - & Abbildung \\
Apr. & - & April \\
Aufl. & - & Auflage \\
bzgl. & - & bezüglich \\
Bd. & - & Band \\
ca. & - & circa \\
conf & - & Konfidenz \\
confmin & - & Mindestkonfidenz \\
D. & - & Deutschland \\
d.h. & - & das heißt \\
EDV & - & elektronische Datenverarbeitung \\
erw. & - & erwarteter \\
et al. & - & et alii \\
etc. & - & et cetera \\
e.V. & - & eingetragener Verein \\
f. & - & folgende \\
FCB & - & First-Choice-Buyer \\
FCV & - & First-Choice-Value \\
Feb. & - & Februar \\
FES & - & Früherkennungssystem \\
ff. & - & fortfolgende \\
FMCG & - & Fast Moving Consumer Goods \\
Gentechn. & - & Gentechnisch \\
Gew. & - & Gewinn \\
GfK & - & Gesellschaft für Konsumforschung \\
Habil. & - & Habilitation \\
Hrsg. & - & Herausgeber \\
i. & - & im \\
i.Allg. & - & Jan Allgemeinen \\
Jan. & - & Januar \\
Jg. & - & Jahrgang \\
\hline
\end{tabular}


Kap.

KDD

klarsp.

KRW

LEH

Lt.

m.

MA

No.

$\mathrm{Nr}$.

o.

öffentl.

p.a.

PLZ

Prod.

rel.

rg.

$\mathrm{S}$.

SGE

sog.

Sp.

sup

sup(präm)

supmin

$\operatorname{supmin}($ präm)

tägl.

TKK

u.

u.a.

u.U.

usw.

v.a.

Verl.
Kapitel

Knowledge Discovery in Databases

Klarspüler

Käuferreichweite

Lebensmitteleinzelhandel

Laut

mit

Marktanteil

Number

Nummer

oder

öffentliche

per annum

Produktlebenszyklus

Produkt

relativer

Reiniger

Seite

Strategische Geschäftseinheit

so genannt

Spalte

Support

Support der Prämisse

Mindestsupport

Mindestsupport der Prämisse

täglich

Tiefkühlkost

und

unter anderem

unter Umständen

und so weiter

vor allem

Verlust 


$\begin{array}{lll}\text { Vgl. } & - & \text { Vergleiche } \\ \text { Vol. } & - & \text { Volume } \\ \text { WiSt } & - & \text { Wirtschaftswissenschaftliches Studium } \\ \text { WiSu } & - & \text { Das Wirtschaftsstudium } \\ \text { WPR } & - & \text { Waschen/Putzen/Reinigen } \\ \text { Z. } & - & \text { Zeitschrift } \\ \text { Z.B. } & - & \text { zum Beispiel } \\ \text { ZFP } & - & \text { Zeitschrift für Forschung und Praxis } \\ \text { ZUMA } & - & \text { Zentrum für Umfragen, Methoden und } \\ & & \text { Analysen }\end{array}$

